\title{
Functional Coiled-Coil-like Assembly by Knob-into-Hole Packing of Single Heptad Repeat
}

Sudipta Mondal,,$\stackrel{+}{\perp}$ Vasantha Basavalingappa, ${ }^{\dagger}$ Guy Jacoby,,+ Linda J. W. Shimon, ${ }^{\S}$ Roy Beck, Ehud Gazit*, *,

†Department of Molecular Microbiology and Biotechnology, George S. Wise Faculty of Life Sciences, Tel Aviv University, Tel Aviv 69978, Israel

\$The Raymond and Beverly Sackler School of Physics and Astronomy, Tel Aviv University, Tel Aviv 69978, Israel

${ }^{\S}$ Department of Chemical Research Support, Weizmann Institute of Science, Rehovot 76100, Israel

"Department of Materials Science and Engineering, Iby and Aladar Fleischman

Faculty of Engineering, Tel Aviv University, Tel Aviv 69978, Israel

\section{Present Address}

$\perp$ (S.M.) Department of Biotechnology, National Institute of Technology, Durgapur 713209, India.

\section{Corresponding Author}

*Email: ehudg@post.tau.ac.il 
a

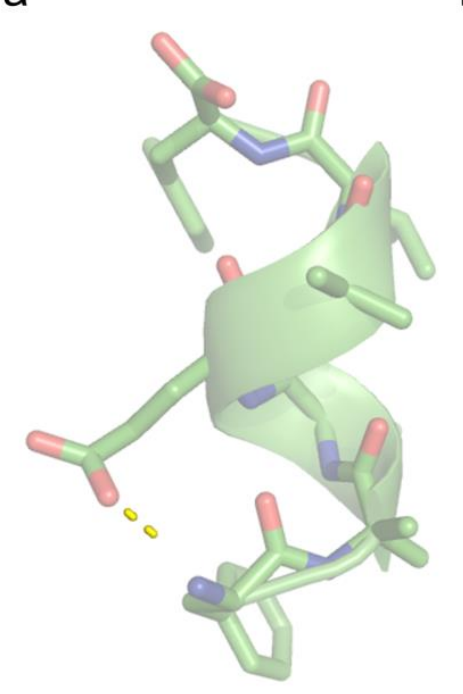

C

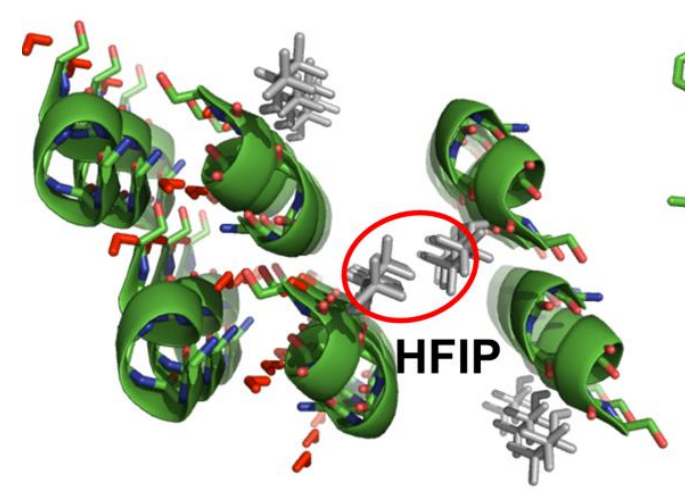

Without side-chain

b
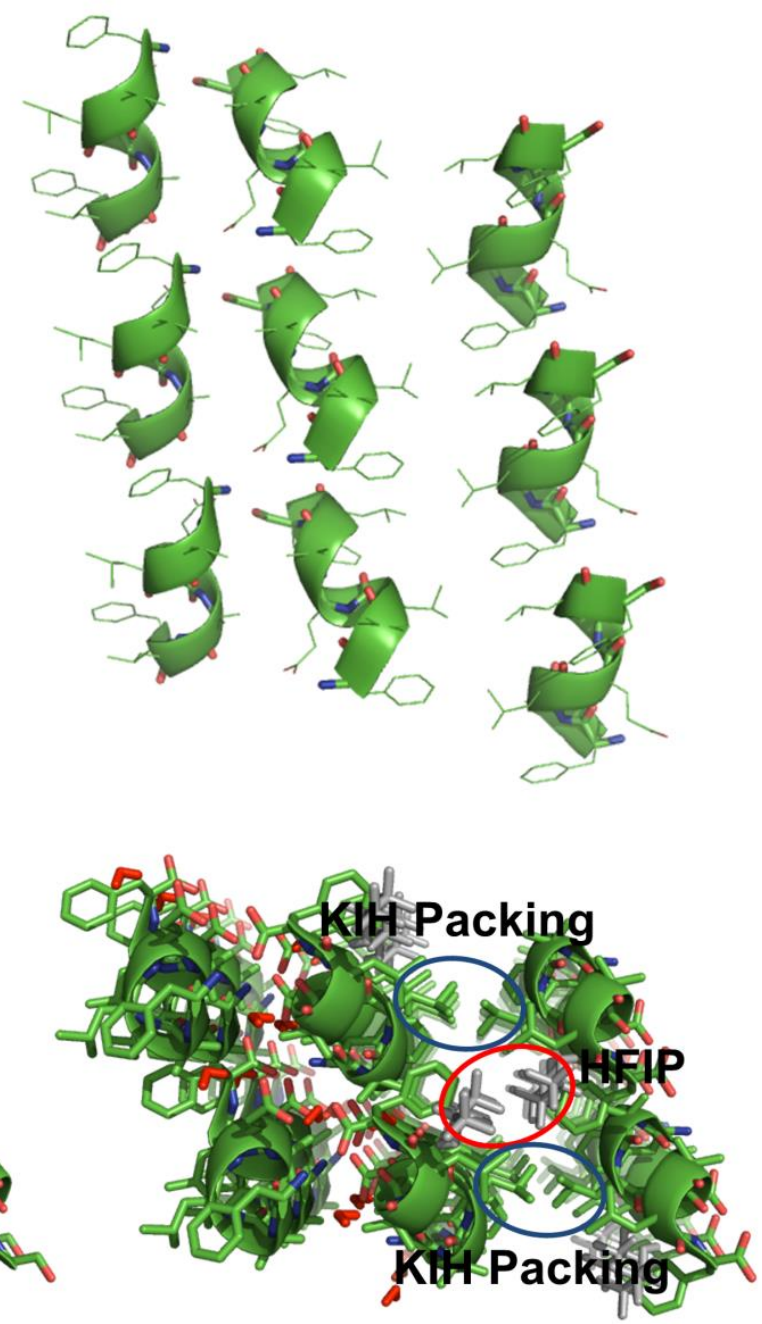

With side-chain

Figure S1. Analysis of $\mathbf{C C}_{\text {SHR }}$ crystal structure. (a) Intramolecular H-bonding between Glu side chain and N-terminal amine in the asymmetric unit. (b) Organization of helical monomers in columnar structures. Three adjacent helical columns were illustrated. Solvents molecules were removed for clarity. (c) Location of the HFIP molecules which stabilized the KIH packed adjacent helical columns through hydrophobic interactions was depicted without (left) or with peptide side chains. 
a

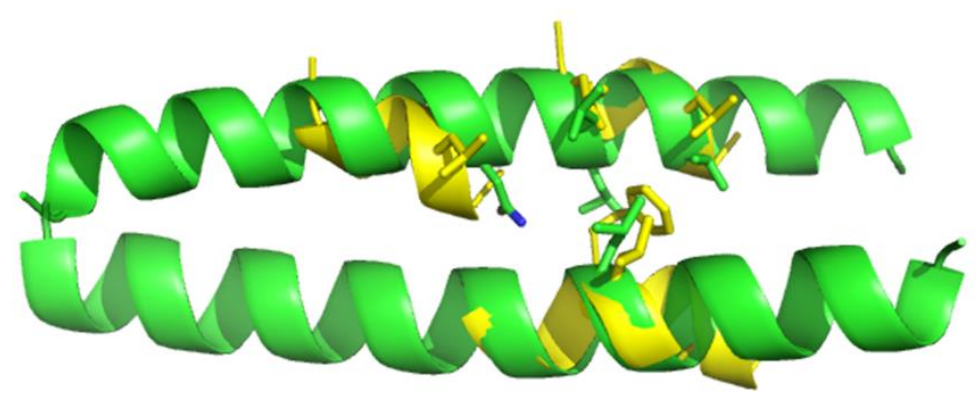

$\mathrm{b}$
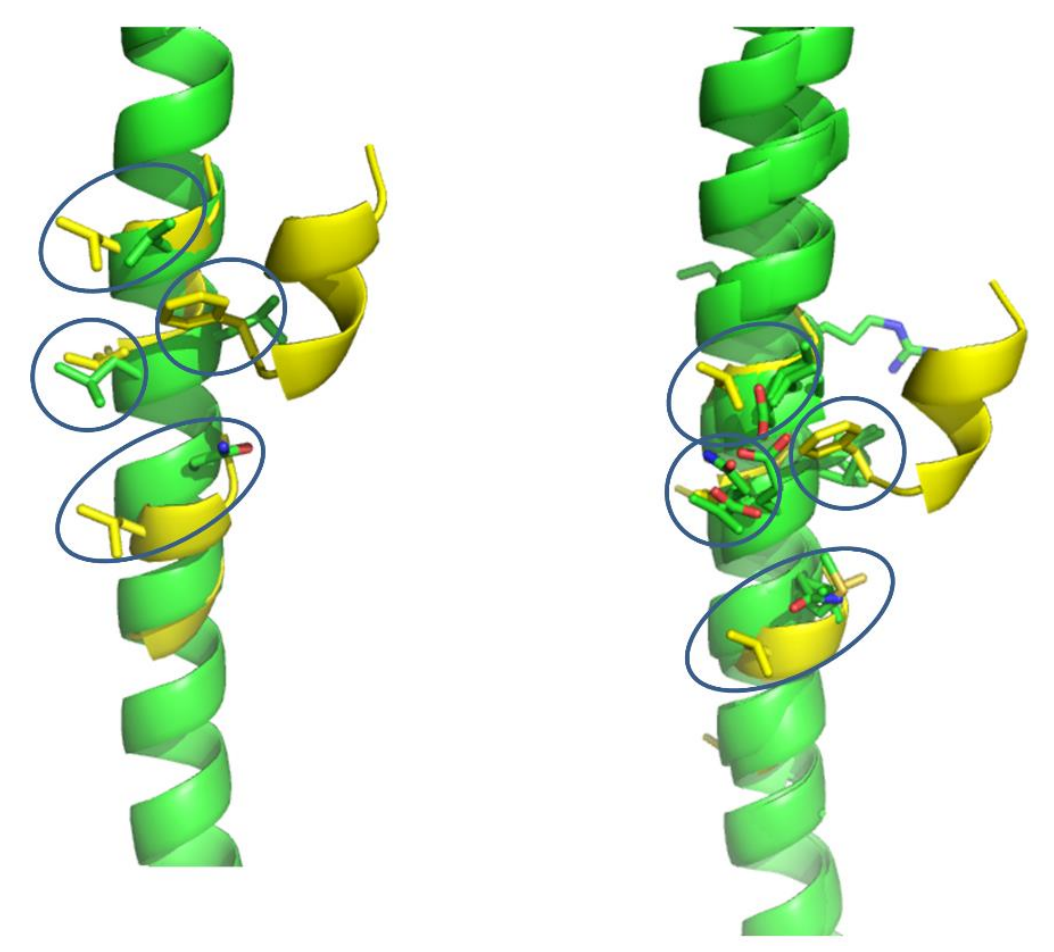

Figure S2. (a) Superposition of GCN4 leucine zipper KIH packing and CCSHR supramolecular KIH packing showing overlap between two structures. (b) Correlation between the hole forming residues of canonical coiled-coil structure (left: pdb id, 2zta; right pdb ids, 1a0a, 2aze, 1pl5, 2zta) and supramolecular KIH packing showed that the hole residues of $\mathrm{CC}_{\mathrm{SHR}}$ are clustered around the hole residues of the canonical KIH motif. 
a

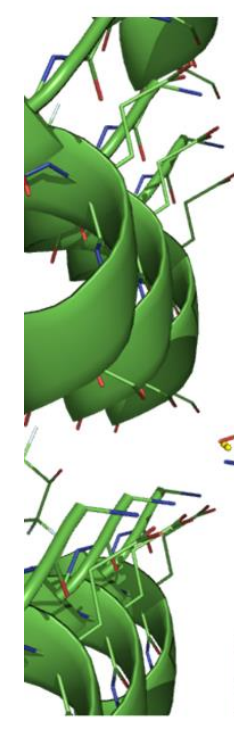

b
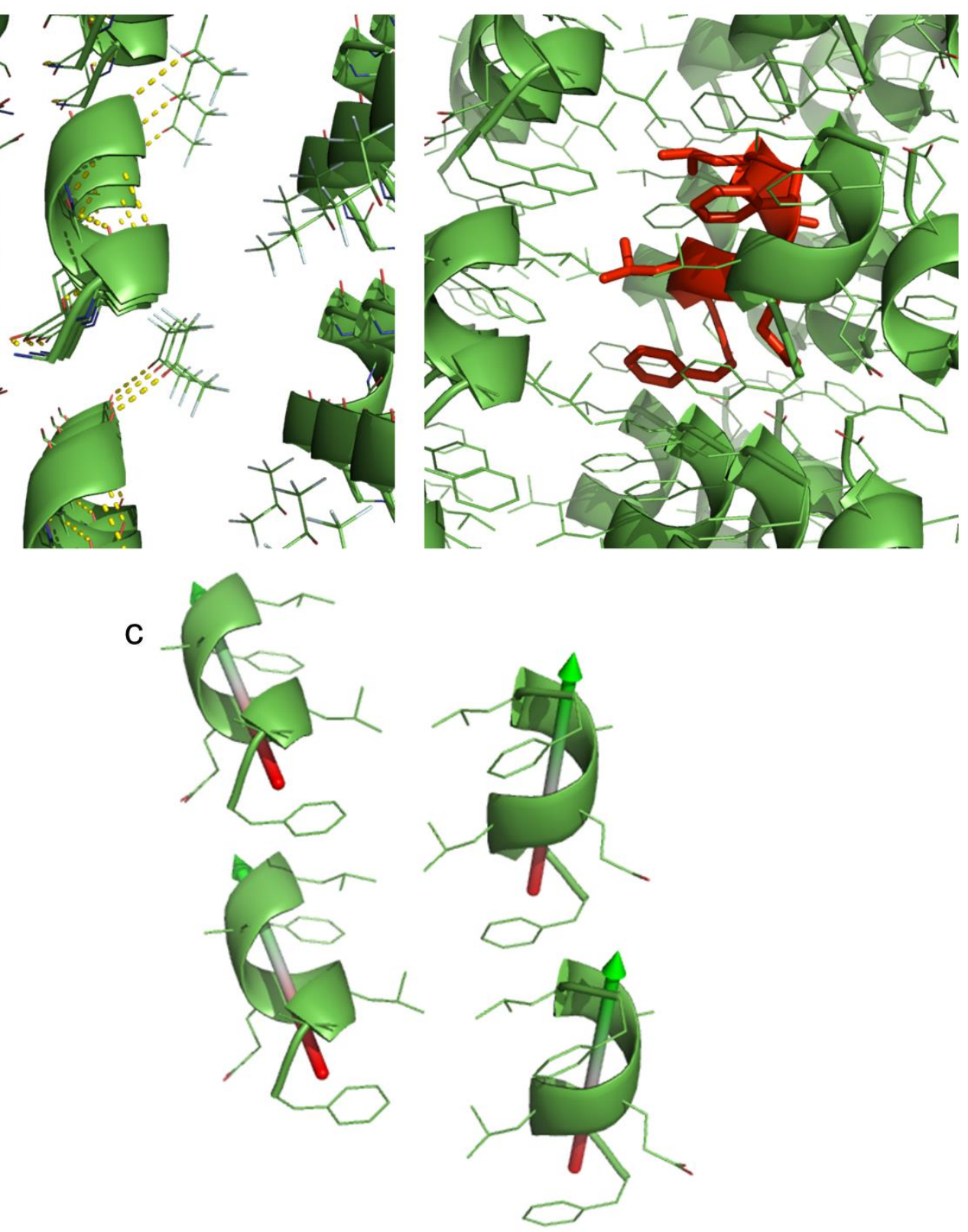

Figure S3. Molecular interactions among helices and their relative alignment. (a)

$\mathrm{H}$-bonding interactions along the longitudinal direction of helical column. Most notably, CCsHR did not form H-bonding between helices positioned on top of each other within a single column. (b) Lack of $\pi$-stacking interactions between adjacent helical columns confirmed the stabilization of the interfaces by KIH packing. Red asymmetric unit was placed to aid in the visualization of the neighboring Phe residues. (c) Representation of helical axis used to determine the angle between helices. 

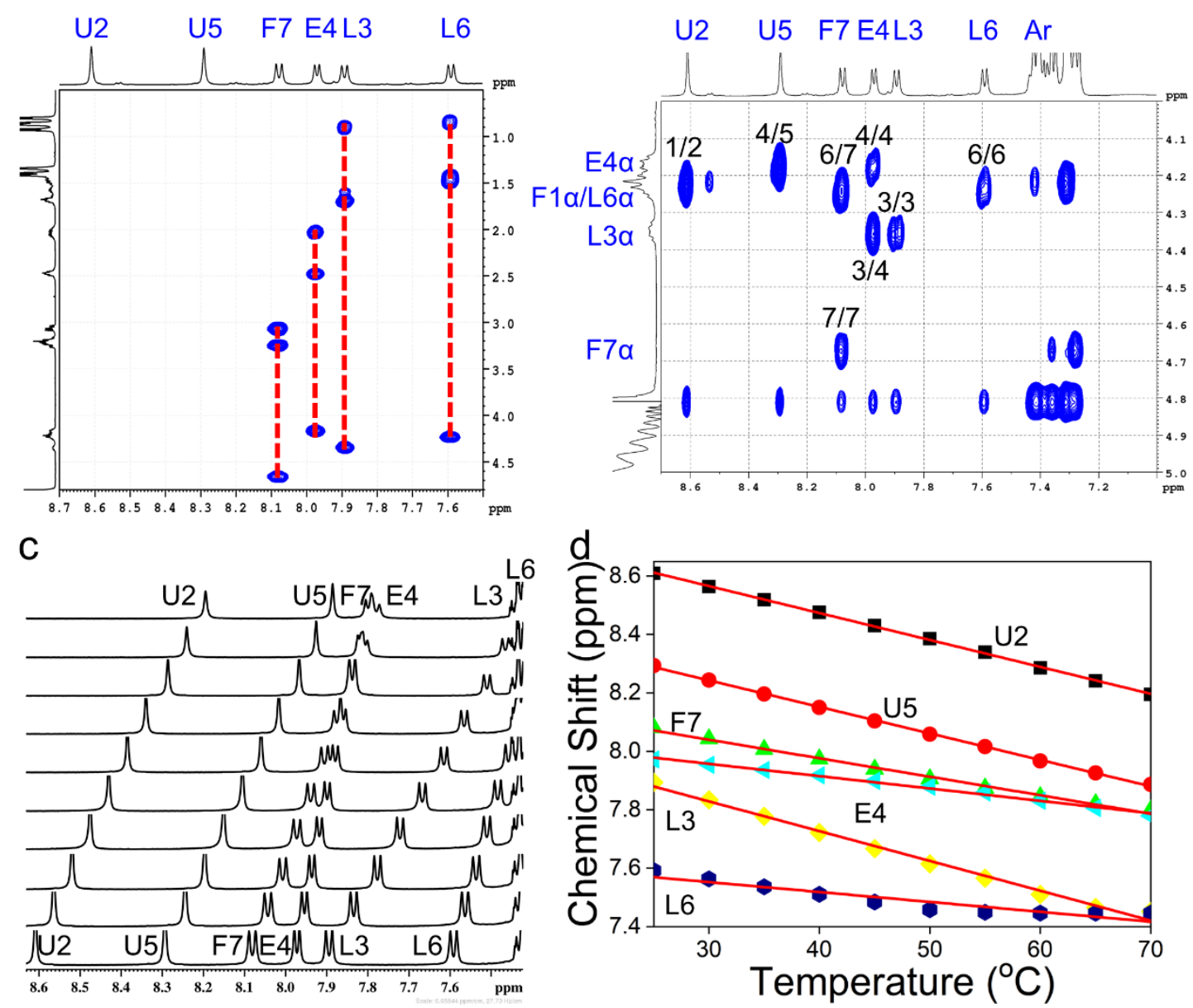

Figure S4. ${ }^{1}$ HNMR characterization of the CC $_{\text {SHR }}$ nanofibers. (a) Partial TOCSY trace indicating residue specific assignments. (b) Finger print NOESY spectral region showed sequential $d_{\alpha \mathrm{N}}$ NOE connectivity. (c) Temperature dependent 1D-NMR spectra. (d) Plot of chemical shift against temperature of amide protons. (One letter notation of the amino acids. Phe, F; Aib, U; Leu, L; Glu, E. Residues are numbered in ascending order from $\mathrm{N}$ - to $\mathrm{C}$-terminus) 

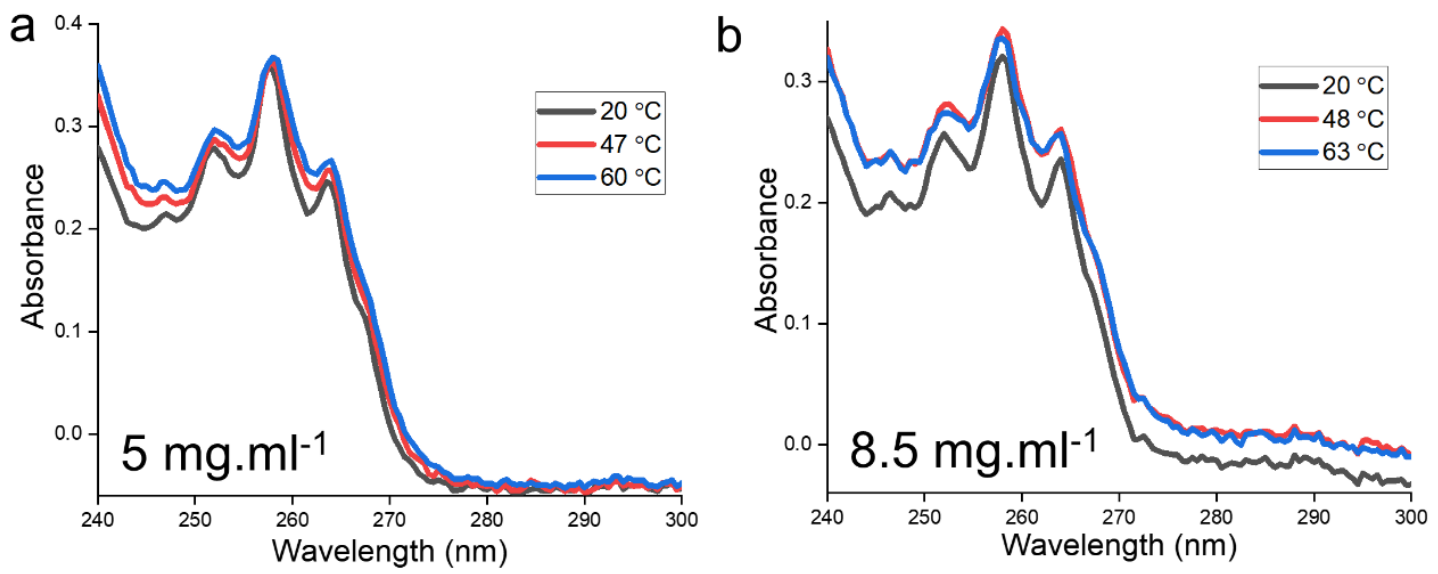

Figure S5. Measurements of temperature dependent optical density of the $\mathrm{CC}_{\mathrm{SHR}}$ nanofibers. (a) Absorbance vs wavelength profile of nanofibers prepared at $5 \mathrm{mg} \mathrm{ml}^{-}$ ${ }^{1}$ concentration at three different temperatures. (b) Absorbance vs wavelength profile of nanofibers prepared at $8.5 \mathrm{mg} \mathrm{ml}^{-1}$ concentration at three different temperatures. 


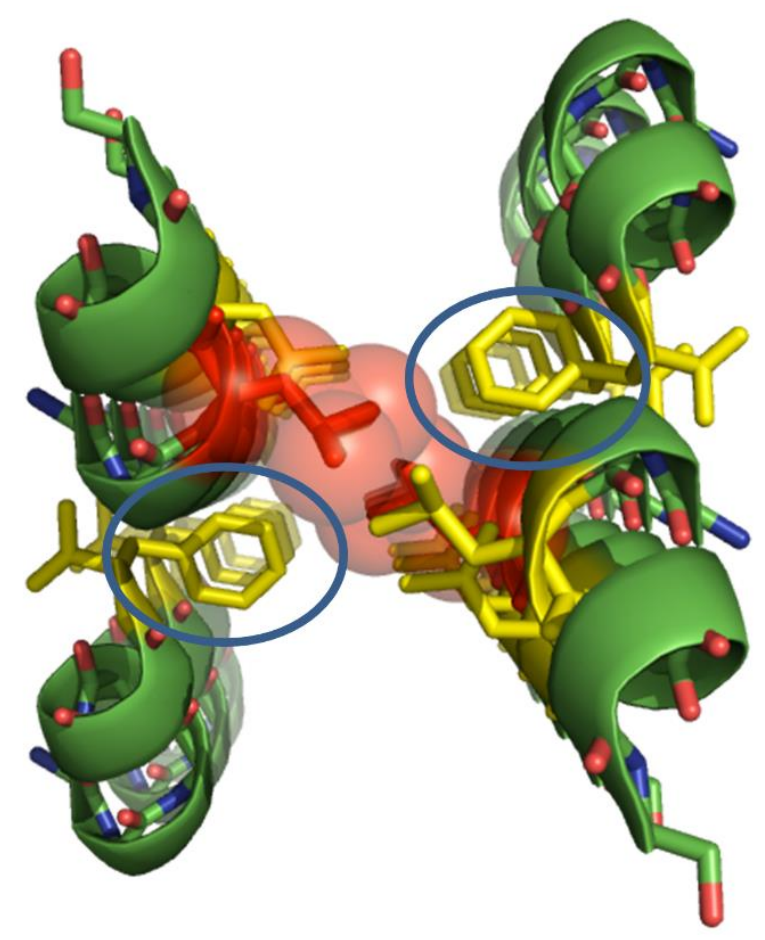

Figure S6: Relative orientation of the phenylalanine residues associated with the supramolecular KIH interaction. 
Table S1. Dihedral angles of different amino acids in CCshr peptide.

\begin{tabular}{|c|c|c|c|}
\hline $\begin{array}{c}\text { Residues (From } \\
\text { C-N terminus) }\end{array}$ & $\boldsymbol{\varphi}$ & $\boldsymbol{\Psi}$ & $\mathbf{I}(\boldsymbol{\varphi}+\boldsymbol{\psi}) \mathbf{I}$ \\
\hline Phe & -96.68 & & \\
\hline Leu & -64.01 & -24.72 & 88.73 \\
\hline Aib & -55.72 & -28.91 & 84.63 \\
\hline Glu & -59.79 & -27.92 & 87.71 \\
\hline Leu & -68.88 & -19.40 & 97 \\
\hline Aib & -46.11 & -51.02 & \\
\hline Phe & 144.48 & & \\
\hline
\end{tabular}


Table S2. Crystal data and structure refinement of $\mathrm{CC}_{\mathrm{SHR}}$.

\begin{tabular}{|c|c|}
\hline Empirical formula & C46 H68 F6 N7 O13 \\
\hline Formula weight & 1041.06 \\
\hline Crystal system & Orthorhombic \\
\hline Space group & $P 22_{1} 2_{1}$ \\
\hline $\mathrm{a}, \AA$ & $10.7269(1)$ \\
\hline $\mathrm{b}, \AA$ & $12.7876(1)$ \\
\hline $\mathrm{c}, \AA$ & $39.6389(3)$ \\
\hline$\alpha^{\circ}$ & 90 \\
\hline$\beta^{\circ}$ & 90 \\
\hline$\gamma^{\circ}$ & 90 \\
\hline $\mathrm{V}\left(\AA^{3}\right)$ & 5437.33(9) \\
\hline $\mathrm{Z}$ & 4 \\
\hline dcalc $\left(\mathrm{mg} / \mathrm{cm}^{3}\right)$ & 1.272 \\
\hline$\mu\left(\mathrm{mm}^{-1}\right)$ & 0.907 \\
\hline Reflections & 98708 \\
\hline Unique reflections/restraints/parameters & $10422 / 4 / 671$ \\
\hline$R_{\text {merge }}$ & 0.0634 \\
\hline $\mathrm{R}[\mathrm{I}>2 \sigma(\mathrm{I})]$ & $\mathrm{R}_{1}=0.0497, \mathrm{wR}_{2}=0.1355$ \\
\hline $\mathrm{R}$ [all] & $\mathrm{R}_{1}=0.0522, \mathrm{wR}_{2}=0.1376$ \\
\hline Goodness of Fit & 1.044 \\
\hline
\end{tabular}

